\title{
Gender and Age Influences in T-Loop Morphology
}

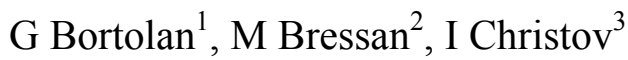 \\ ${ }^{1}$ Institute of Biomedical Engineering ISIB-CNR, Padova, Italy \\ ${ }^{2}$ Cardiology Dept., University of Padova, Italy \\ ${ }^{3}$ Centre of Biomedical Engineering, Bulgarian Academy of Sciences, Sofia, Bulgaria
}

\begin{abstract}
In the present study the influence of gender and age on the T-loop morphology is taken into account. The population based ECG-ILSA database (Italian Longitudinal Study on Aging) was used. The patient, classified as healthy (328), with cardiac diseases (172), and with hypertension (643) were included in this study. For this purpose, ten parameters characterizing $T$ wave morphology were obtained from the VCG. In addition to the two gender groups, two age groups have been established $(<75$ years and $\geq 75$ years old $)$.

From this study, it turns out that age and gender influence the T-loop morphology differently in the three considered groups. The gender influences the healthy and patients with hypertension, while age influences mainly the patients with cardiac diseases.
\end{abstract}

\section{Introduction}

In the last years there is an interest in the analysis of the repolarization phase, and several recent publications aroused the interest in T-wave morphology [1-4]

The aim of this study is to investigate influence of gender and age on the T-loop morphology. This study is a continuation of the investigation performed in [5], in which the authors studied the gender and age influences in QT-dispersion. For this purpose, the same population based ECG database from the longitudinal ILSA study has been used. Three groups of patients have been considered: healthy subjects, patients with cardiac disease and with hypertension. Five parameters obtained from the synthesized VCG were computed with two methods, and a validation study was performed.

\section{Material and methods}

Particular aspects of T-wave morphology are considered for characterization and quantification of heterogeneous repolarization, using the vectorcardiogram
(VCG). The orthogonal Frank leads were synthesized from the standard 12 lead ECG $[6,7]$.

Five parameters were obtained from the VCG [8]:

- maximum angle between QRS and T loop axes (MA)

- $\quad \mathrm{T}$ axis elevation and azimuth angle difference (DEA)

- $\quad$ Ratio of maximum to mean $\mathrm{T}$ vector magnitudes (RMMV)

- $\quad$ Angle of the T-loop in frontal plane (TF)

- $\quad$ Angle of the T-loop in the horizontal plane (TH).

In addition, as described in [8], a modified computation method was performed. The reference zero point of the VCG loop was chosen appropriately (zero*) between the two points of the loop with the maximum distance. Then five modified parameters (MAm, DEAm, RMMVm ,TFm, THm) are measured with respect to the new considered zero* point.

\subsection{Experimental data}

The population based ECG-ILSA database (Italian Longitudinal Study on Aging) has been considered. This database is composed by 2513 ECG signals of elderly people (1337 males and 1176 females aged from 65 to 85 years old) [9-11].

The following three groups have been selected for this study:

- 328 healthy subjects

- 643 patients with only hypertension (HYPT)

- 172 patients with only cardiac diseases (CARD)

The three groups are mutually exclusive. The "healthy" group is characterized by absence of cardiovascular and chronic pulmonary diseases, by no use of drugs that can influence the electrical cardiac activity, and by no electrolyte imbalance [9].

In addition to the two gender groups, two age groups have been established ( $<75$ years and $\geq 75$ years old). 
Table 1. Mean value \pm standard deviation of the parameters MA, DEA, RMMV, TF, TH and MAm, DEAm, RMMVm, TFm, THm in Male and Female in the Healthy group.

\begin{tabular}{lccc}
\hline Parameter & $\mathrm{M}$ & $\mathrm{F}$ & $\mathrm{p}$ \\
\hline MA & $87.8 \pm 52.3$ & $67.3 \pm 50.0$ & $\mathrm{p}<0.001$ \\
DEA & $62.7 \pm 17.0$ & $54.8 \pm 16.0$ & $\mathrm{p}<0.001$ \\
RMMV & $2.2 \pm 0.3$ & $2.2 \pm 0.3$ & n.s. \\
TF & $33.9 \pm 17.3$ & $38.6 \pm 13.2$ & $\mathrm{p}<0.01$ \\
TH & $-23.5 \pm 24.0$ & $-8.9 \pm 17.9$ & $\mathrm{p}<0.001$ \\
MAm & $91.6 \pm 55.5$ & $69.5 \pm 52.0$ & $\mathrm{p}<0.001$ \\
DEAm & $57.2 \pm 18.5$ & $56.1 \pm 20.4$ & n.s. \\
RMMVm & $2.4 \pm 0.3$ & $2.4 \pm 0.3$ & n.s. \\
TFm & $33.0 \pm 19.3$ & $37.8 \pm 15.4$ & $\mathrm{p}=0.017$ \\
THm & $-21.6 \pm 27.8$ & $-5.6 \pm 22.6$ & $\mathrm{p}<0.001$ \\
\hline
\end{tabular}

Table 2. Mean value \pm standard deviation of the parameters MA, DEA, RMMV, TF, TH and MAm, DEAm, RMMVm, TFm, THm in $M$ and $F$ in the CARD group.

\begin{tabular}{lccl}
\hline Parameter & $\mathrm{M}$ & $\mathrm{F}$ & $\mathrm{p}$ \\
\hline MA & $100.9 \pm 52.4$ & $92.1 \pm 54.9$ & n.s. \\
DEA & $62.3 \pm 1.8$ & $60.3 \pm 20.1$ & n.s. \\
RMMV & $2.1 \pm 0.4$ & $2.1 \pm 0.5$ & n.s. \\
TF & $25.1 \pm 53.4$ & $30.6 \pm 50.7$ & n.s. \\
TH & $-30.9 \pm 48.9$ & $-26.3 \pm 45.1$ & n.s. \\
MAm & $106.7 \pm 57.4$ & $97.6 \pm 59.7$ & n.s. \\
DEAm & $58.4 \pm 23.6$ & $64.8 \pm 22.6$ & n.s. \\
RMMVm & $2.2 \pm 0.4$ & $2.2 \pm 0.5$ & n.s. \\
TFm & $22.8 \pm 57.5$ & $25.3 \pm 51.8$ & n.s. \\
THm & $-29.3 \pm 54.7$ & $-20.1 \pm 56.8$ & n.s. \\
\hline
\end{tabular}

Table 3. Mean value \pm standard deviation of the parameters MA, DEA, RMMV, TF, TH and MAm, DEAm, RMMVm, TFm, THm in M and F in the HYPT group.

\begin{tabular}{lccc}
\hline Parameter & $\mathrm{M}$ & $\mathrm{F}$ & $\mathrm{p}$ \\
\hline MA & $96.2 \pm 52.2$ & $82.9 \pm 51.3$ & $\mathrm{p}<0.001$ \\
DEA & $62.1 \pm 15.1$ & $57.2 \pm 19.5$ & $\mathrm{p}<0.001$ \\
RMMV & $2.2 \pm 0.3$ & $2.1 \pm 0.4$ & $\mathrm{p}=0.022$ \\
TF & $32.4 \pm 28.5$ & $38.2 \pm 35.1$ & $\mathrm{p}=0.024$ \\
TH & $-23.0 \pm 29.4$ & $-13.0 \pm 37.6$ & $\mathrm{p}<0.001$ \\
MAm & $102.1 \pm 55.3$ & $84.9 \pm 55.4$ & $\mathrm{p}<0.001$ \\
DEAm & $59.9 \pm 19.9$ & $57.6 \pm 21.2$ & n.s. \\
RMMVm & $2.3 \pm 0.3$ & $2.3 \pm 0.4$ & n.s. \\
TFm & $31.1 \pm 32.9$ & $35.9 \pm 37.4$ & n.s. \\
THm & $-22.9 \pm 34.7$ & $-9.1 \pm 40.5$ & $\mathrm{p}<0.001$ \\
\hline
\end{tabular}

Table 4. Mean value \pm standard deviation of the parameters MA, DEA, RMMV, TF, TH and MAm, DEAm, RMMVm, TFm, THm in $<75$ and $\geq 75$ in the Healthy group.

\begin{tabular}{lccl}
\hline Parameter & $<75$ & $\geq 75$ & $\mathrm{p}$ \\
\hline MA & $78.4 \pm 51.8$ & $80.1 \pm 53.3$ & n.s. \\
DEA & $58.8 \pm 17.4$ & $60.2 \pm 16.3$ & n.s. \\
RMMV & $2.2 \pm 0.3$ & $2.2 \pm 0.3$ & n.s. \\
TF & $35.7 \pm 15.0$ & $36.4 \pm 17.5$ & n.s. \\
TH & $-17.5 \pm 24.1$ & $-16.5 \pm 19.6$ & n.s. \\
MAm & $80.0 \pm 54.6$ & $86.4 \pm 55.8$ & n.s. \\
DEAm & $56.2 \pm 18.9$ & $57.7 \pm 20.2$ & n.s. \\
RMMVm & $2.3 \pm 0.3$ & $2.4 \pm 0.3$ & n.s. \\
TFm & $34.8 \pm 16.0$ & $35.5 \pm 21.2$ & n.s. \\
THm & $-15.0 \pm 28.7$ & $-14.1 \pm 22.5$ & n.s. \\
\hline
\end{tabular}


Table 5. Mean value \pm standard deviation of the parameters MA, DEA, RMMV, TF, TH and MAm, DEAm, RMMVm, TFm, THm in $<75$ and $\geq 75$ in the CARD group.

\begin{tabular}{lccc}
\hline Parameter & $<75$ & $\geq 75$ & $\mathrm{p}$ \\
\hline MA & $88.0 \pm 51.4$ & $109.5 \pm 53.6$ & $\mathrm{p}<0.01$ \\
DEA & $61.6 \pm 18.2$ & $61.6 \pm 20.5$ & n.s. \\
RMMV & $2.1 \pm 0.4$ & $2.1 \pm 0.5$ & n.s. \\
TF & $27.9 \pm 47.7$ & $26.2 \pm 57.8$ & n.s. \\
TH & $-22.9 \pm 44.7$ & $-36.9 \pm 49.8$ & n.s. \\
MAm & $90.4 \pm 56.6$ & $119.3 \pm 56.6$ & p=0.001 \\
DEAm & $60.0 \pm 21.2$ & $61.8 \pm 26.0$ & n.s. \\
RMMVm & $2.2 \pm 0.4$ & $2.2 \pm 0.5$ & n.s. \\
TFm & $26.1 \pm 51.8$ & $20.7 \pm 59.6$ & n.s. \\
THm & $-19.7 \pm 51.1$ & $-33.4 \pm 60.0$ & n.s. \\
\hline
\end{tabular}

Table 6. Mean value \pm standard deviation of the parameters MA, DEA, RMMV, TF, TH and MAm, DEAm, RMMVm, TFm, THm in $<75$ and $\geq 75$ in the HYPT group.

\begin{tabular}{lccl}
\hline Parameter & $<75$ & $\geq 75$ & $\mathrm{p}$ \\
\hline MA & $87.9 \pm 51.5$ & $90.3 \pm 53.2$ & n.s. \\
DEA & $59.4 \pm 17.3$ & $59.4 \pm 18.8$ & n.s. \\
RMMV & $2.1 \pm 0.4$ & $2.1 \pm 0.3$ & n.s. \\
TF & $35.4 \pm 29.2$ & $36.1 \pm 38.0$ & n.s. \\
TH & $-15.3 \pm 33.3$ & $-21.4 \pm 36.4$ & p=0.031 \\
MAm & $92.1 \pm 55.5$ & $93.2 \pm 57.0$ & n.s. \\
DEAm & $59.2 \pm 20.5$ & $57.5 \pm 20.9$ & n.s. \\
RMMVm & $2.3 \pm 0.4$ & $2.3 \pm 0.3$ & n.s. \\
TFm & $34.2 \pm 32.1$ & $32.9 \pm 41.4$ & n.s. \\
THm & $-13.2 \pm 36.9$ & $-18.9 \pm 41.6$ & n.s. \\
\hline
\end{tabular}

Table 7. Summary of $p$ values of the paired t-test of the parameters MA, DEA, RMMV, TF, TH and MAm, DEAm, RMMVm, TFm, THm in considering the Healthy, CARD, HYPT groups (s.1. 5\%).

\begin{tabular}{lcccccc}
\hline & \multicolumn{2}{c}{ Healthy } & \multicolumn{2}{c}{ Card } & \multicolumn{2}{c}{ Hytp } \\
\hline Parameter & M-F & age & M-F & age & M-F & age \\
\hline MA & $<.01$ & n.s. & n.s. & $<.01$ & $<.01$ & n.s. \\
DEA & $<.01$ & n.s. & n.s. & n.s. & $<.01$ & n.s. \\
RMMV & n.s. & n.s. & n.s. & n.s. & .02 & n.s. \\
TF & $<.01$ & n.s. & n.s. & n.s. & .02 & n.s. \\
TH & $<.01$ & n.s. & n.s. & n.s. & $<.01$ & .03 \\
MAm & $<.01$ & n.s. & n.s. & $<.01$ & $<.01$ & n.s. \\
DEAm & n.s. & n.s. & n.s. & n.s. & n.s. & n.s. \\
RMMVm & n.s. & n.s. & n.s. & n.s. & n.s. & n.s. \\
TFm & .022 & n.s. & n.s. & n.s. & n.s. & n.s. \\
THm & $<.01$ & n.s. & n.s. & n.s. & $<.01$ & n.s. \\
\hline
\end{tabular}

\section{Results and discussion}

The 5 'original' parameters (MA, DEA, RMMV, TF, $\mathrm{TH}$ ) and the 5 modified parameters (MAm, DEAm, RMMVm, TFm, THm) have been computed in the 3 groups of patients selected from the ECG-ILSA database.

First, the gender influence has been investigated. Table 1 reports the mean value and the standard deviation of the considered parameters in Male and Female in the healthy group and the p-value of the t-test. Table 2 and Table 3 report respectively the corresponding results in the CARD and HYPT groups.

Analysing these tables, it is possible to observe that age influences only the healthy and the HYPT groups. For example, the MA index in the healthy group shows the values of $87.8 \pm 52.3$ in male and $67.3 \pm 50.0$ in female $(p<0,001)$; in the hypertension group $96.2 \pm 52.2$ in $\mathrm{M}$ and $82.7 \pm 51.3$ in $\mathrm{F}(\mathrm{p}=0,001)$, while in patients with cardiac disease no significant differences were observed. The remaining 9 parameters show "substantially" a similar behaviour.

The subsequent analysis consider the two age subgroups ( $<75$ years and $\geq 75$ years old). Table 4 reports the mean value and the standard deviation of the considered parameters in $<75$ and $\geq 75$ in the healthy group and the $\mathrm{p}$-value of the t-test. 
Table 5 and Table 6 report respectively the corresponding results in the CARD and HYPT groups.

Considering the MA index in the two age subgroups, the following results were respectively observed: no significant differences in the healthy group and in the hypertension group, while $88.0 \pm 51.4$ ( $<75$ years old) and $109.5 \pm 53.6$ ( $\geq 75$ years old) in patients with cardiac diseases $(\mathrm{p}<0,01)$.

Table 7 report the summary of all the p-value of the ttest of the previous Tables 1-6.

These results are in agreement with the results obtained in [5], where QT-dispersion indices are influenced in the healthy group by gender, in the group of subjects with cardiac disease by age, while in the hypertension group by age and gender.

From this study, it turns out that age and gender influence the T-loop morphology differently in the three considered groups.

Considering that ventricular repolarization can be differently modified by various pathologies, further investigations are needed to study the clinical meaning of this differentiated behaviour of T-loop morphology and QT dispersion.

\section{Acknowledgements}

The authors acknowledge the Italian Longitudinal Study on Aging project, ILSA (Targeted Project of the National Research Council of Italy on Aging), and the ILSA Working Group: Luigi Amaducci, Guglielmo Scarlato, Marzia Baldereschi, Livia Candelise, Antonio Di Carlo, Francesco Grigoletto, Stefania Maggi, Nadia Minicuci, Elio Scarpini, Giuseppina Volonnino, Giovanni Bortolan, Marta Bressan, Carlo Loeb, Carlo Gandolfo, Nicola Canal, Massimo Franceschi, Leontino Battistin, Giuliano Enzi, Augusto Ghetti, Domenico Inzitari, Rossano Vergassola, Salvatore Bonaiuto, Franco Rengo, Antonio Capurso, Paolo Livrea, Luciano Motta.

\section{References}

[1] Lemire D, Pharand C, Rajaonah J, Dube B, LeBlanc A. Wavelet time entropy, $\mathrm{T}$ wave morphology and myocardial ischemia. IEEE Trans. Biomedical Engineering 2000;47:967-970.
[2] Okin P, Devereux R, Fabsitz R, Lee E, Galloway J, Howard B. Principal component analysis of $\mathrm{T}$ wave and prediction of cardiovascular mortality in American Indians. Circulation 2002;105:714-719.

[3] Zabel M, Malik M. Practical use of $T$ wave morphology assessment. Card Electrophysiol Rev 2002;6(3):316-22.

[4] Bortolan G, Bresan M, Christov I. Longitudinal modifications of T-loop morphology. Computers in Cardiology 2002; 29: 685-688.

[5] Bortolan G, Bressan M, Golferini F, for the ILSA research group. Age and gender influences in QT dispersion. Computers in Cardiology 2000; 27: 359362.

[6] Levkov C. Orthogonal electrocardiogram derived from the limb and chest electrodes of the conventional 12-lead system. Med. \& Biol. Eng. \& Comp. 1987;25:155-164.

[7] Daskalov I, Dotsinsky I, Christov I. Developments in ECG acquisition, preprocessing, parameter measurement and recording. IEEE Eng. in Med. \& Biol. 1998; 17:50-58.

[8] Bortolan G, Christov I. Myocardial infarction and ischemia characterization from T-loop morphology in VCG. Computers in Cardiology 2001; 28: 633636.

[9] Bressan M, Bortolan G, Cavaggion C, Fusaro S, for the ILSA Group. Normal ECG in the elderly (the ILSA study) (in Italian). Giornale Italiano di Cardiologia 1998;28:22-28.

[10] Bortolan G, Bressan M, Cavaggion C, Fusaro S, for the ILSA Research Group. ECG analysis in the Italian Longitudinal Study on Aging (ILSA). In: Murray A, Arzbaecher R, eds. Computers in Cardiology 95. Los Alamitos, CA: IEEE Computer Society, 1995: 409-412.

[11] Maggi S, Zucchetto M, Grigoletto F, Baldereschi M, Candelise L, Scarpini E, Scarlato G, Amaducci L, for the ILSA Group. The Italian Longitudinal Study on Aging (ILSA): design and methods. Aging Clin. Exp. Res. 1994;6:464-473.

Address for correspondence.

Giovanni Bortolan

ISIB-CNR

Corso Stati Uniti, 4

35127 Padova , Italy

e-mail: bortolan@isib.cnr.it

www.isib.cnr.it/Research/ISIB_cardio.html 